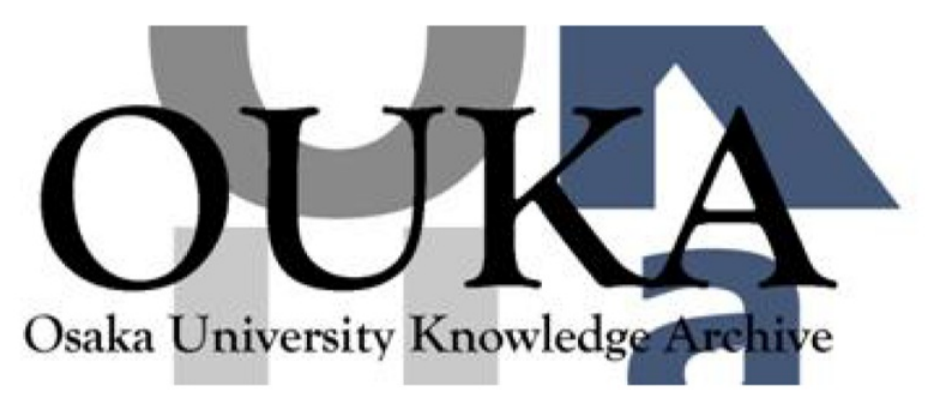

\begin{tabular}{|c|c|}
\hline Title & $\begin{array}{l}\text { Imaging defects in a plate with complex } \\
\text { geometries }\end{array}$ \\
\hline Author(s) & Hayashi, Takahiro \\
\hline Citation & Applied Physics Letters. 108(8) p.081901 \\
\hline Issue Date & $2016-02-22$ \\
\hline oaire:version & VoR \\
\hline URL & https://hdl. handle. net/11094/84487 \\
\hline rights & $\begin{array}{l}\text { This article may be downloaded for personal use } \\
\text { only. Any other use requires prior permission } \\
\text { of the author and AIP Publishing. This article } \\
\text { appeared in Applied Physics Letters and may be } \\
\text { found at https://doi.org/10.1063/1.4942599. }\end{array}$ \\
\hline Note & \\
\hline
\end{tabular}

Osaka University Knowledge Archive : OUKA

https://ir. Library. osaka-u. ac. jp/

Osaka University 


\section{Imaging defects in a plate with complex geometries}

Cite as: Appl. Phys. Lett. 108, 081901 (2016); https://doi.org/10.1063/1.4942599

Submitted: 23 December 2015 . Accepted: 08 February 2016 . Published Online: 23 February 2016

Takahiro Hayashi

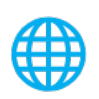

View Online

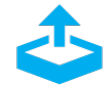

\section{ARTICLES YOU MAY BE INTERESTED IN}

Defect imaging for plate-like structures using diffuse field

The Journal of the Acoustical Society of America 143, EL260 (2018); https://

doi.org/10.1121/1.5030915

Vibration energy analysis of a plate for defect imaging with a scanning laser source technique The Journal of the Acoustical Society of America 140, 2427 (2016); https://

doi.org/10.1121/1.4964275

Rapid thickness measurements using guided waves from a scanning laser source

The Journal of the Acoustical Society of America 126, 1101 (2009); https://

doi.org/10.1121/1.3177268

\section{Challenge us.}

What are your needs for periodic signal detection?

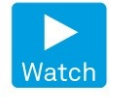

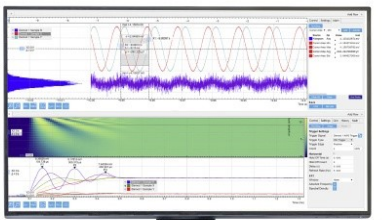

-
- Zurich

- Instruments 


\title{
Imaging defects in a plate with complex geometries
}

\author{
Takahiro Hayashi ${ }^{\text {a) }}$ \\ Graduate School of Engineering, Kyoto University, Kyoto 615-8540, Japan
}

(Received 23 December 2015; accepted 8 February 2016; published online 23 February 2016)

\begin{abstract}
This paper discusses a defect imaging technique using signals containing multiple reflected waves, for a plate with complex geometries, in which direct waves from a generating point to a receiving point cannot be measured. This technique utilizes a characteristic of flexural vibrations wherein the amplitude of the received signals is approximately inversely proportional to the thickness of the source of the elastic wave when a laser pulse is applied to the surface of the plate. First, a theoretical modal analysis elucidated that the amplitude distributions obtained by scanning the laser source of the elastic waves consist of thickness distributions and spurious distributions caused by multiple reflections in the plate. The amplitude distributions were then experimentally obtained for a complex-shaped plate with an artificial defect. The distributions were found to consist of a defect image as well as spurious images, and this agreed well with the theoretical discussions. Clearer defect images with small spurious images were obtained even for a plate with complex geometries by taking an average of the images obtained at multiple frequencies. (C) 2016 AIP Publishing LLC.
\end{abstract}

[http://dx.doi.org/10.1063/1.4942599]

Ultrasonic defect identification techniques are widely used for the non-destructive evaluation of structures. ${ }^{1}$ In the most popular and basic ultrasonic pulse echo method, defects are located using the arrival time of an echo signal and the speed of the waves in the material. The synthetic aperture focusing technique (SAFT) ${ }^{2,3}$ and phased array, ${ }^{4}$ in which several ultrasonic sensor elements transmit and receive signals which are then processed to create defect images, have also became popular techniques. Waves travelling along a straight path between ultrasonic transducers and reflective objects such as defects are generally used to create such defect images, and waves that are reflected and refracted from the edges of the object and resonant vibrations in the object are not considered in these techniques. In particular, in lowfrequency ultrasonic inspections such as guided-wave testing, ${ }^{5-7}$ we often encounter the problem of it not being possible to separate the direct waves and reflected waves, and therefore defects cannot be identified. In such cases, low-frequency vibrations resonate in the object and defects can be detected by measuring the shift in the resonant frequencies. For example, in facility diagnosis, we often use impact hammer testing, in which audible sound is generated by impacting the object with a hammer and the object is evaluated from the resonant frequencies of the sound. Resonant spectroscopy in the ultrasonic range has also been studied. ${ }^{8}$ Ogi et al. ${ }^{9}$ successfully measured the elastic-stiffness coefficients of thin films using non-contact resonant ultrasonic spectroscopy with electromagnetic acoustic transducers. However, resonant spectroscopy generally evaluates characteristics of a whole vibration region and cannot be used for defect location and imaging.

Recently, topological sensitivity analysis ${ }^{10-16}$ has been developed as an effective means of defect imaging based on signals originating from multiple reflections from an object. In the imaging method, wave fields for time-reversed incident waves from array sensors in objects with and without

${ }^{\text {a)} E l e c t r o n i c ~ m a i l: ~ h a y a s h i @ k u a e r o . k y o t o-u . a c . j p . ~ T e l . / F a x: ~+81-75-383-3797 . ~}$ defects are calculated first, and then the space function obtained from the difference of the wave fields gives the defect images. ${ }^{10,11}$ Rodriguez et al. created images of artificial defects in bulky media and plates by accelerating the calculation speed in the topological imaging. ${ }^{12}$ They also proposed the application of topological imaging to defect imaging in a complex geometry where there is no straight path from an ultrasonic transducer to a particular region of interest. ${ }^{13}$ Defect images of the region were obtained by measuring the wave fields of an intact medium in advance and by detecting echo signals at a single transducer location in the inspected object. ${ }^{14}$ Although topological imaging can create defect images of an object with complex geometries at a single transducer location, measurements in a reference medium of the same shape and with the same boundary conditions except for the defects are required, which limits its application to practical non-destructive evaluation. This paper describes a simple defect imaging technique for a plate with complex geometries without either reference media measurements or the calculation of wave fields.

A previous study developed a defect imaging technique in which a flexural A0 mode of Lamb waves generated by laser pulse applied to a thin plate is measured. ${ }^{17}$ In this study, the hybrid calculation technique combining the semianalytical finite element and finite element methods revealed that the amplitude in the A0 mode is approximately inversely proportional to the thickness of the laser spot; this is effective for a plate with rounded defects in the low-frequency range below the A1 cut-off frequency. Moreover, two-dimensional images of the thickness distribution were obtained by application of the scanning laser source technique with a fixed ultrasonic receiving transducer. Then, the study used calculations and experiments to prove that defect images could be obtained even in a plate with square notches, with these images becoming clearer in the lower frequency thickness product $(f d)$ range. ${ }^{18}$ The use of a low $f d$ range enabled us to use aircoupled ultrasonic sensors instead of contact ultrasonic 
transducers as receivers and thus realize a completely noncontact defect imaging technique. ${ }^{19}$ The defect imaging techniques utilize a characteristic of the flexural vibrations wherein relatively large flexural vibrations are generated when a laser beam is emitted in the vicinity of or on a defect which is a thin area in a plate. In these studies, the direct waves propagating in a straight path between laser emission spots and receiving transducers were mainly used for defect imaging. However, considering the characteristic whereby relatively large flexural vibrations are generated in defective areas by laser emission, we can infer that the imaging technique can also be applied to plates with complex geometries where only refracted and reflected waves are measured. To prove this, theoretical discussions using modal analysis of finite element modeling and experimental results are shown below.

Next, we consider a procedure in which a laser spot $\mathbf{x}_{S}$ for generating elastic waves is scanned over a plate and in which the elastic waves are received at $\mathbf{x}_{R}$ by a sensor fixed on the plate, as shown in Fig. 1. There is no straight path between the laser spots and the receiving sensor, and only the reflected and refracted waves can be detected. In such a case, the received waveforms are affected by resonant modes of the plate due to there being multiple reflections at the plate edges as well as the plate thickness at the laser spots. To clarify the relationship between the amplitude distribution and resonant mode, we used modal analysis of finite element modeling for a plate at a harmonic vibration with an angular frequency $\omega$.

In finite element modeling where a closed region is divided into small elements, the nodal displacement vector $\mathbf{u}$ is governed by the following equation of motion for a harmonic vibration $\exp (i \omega t)$ with angular frequency $\omega$.

$$
-\omega^{2} \mathbf{M u}+i \omega \mathbf{C u}+\mathbf{K u}=\mathbf{f},
$$

where the mass matrix $\mathbf{M}$, damping matrix $\mathbf{C}$, and kinetic matrix $\mathbf{K}$ are obtained from material constants and geometries, and the external force vector $\mathbf{f}$ is determined by the external loading. For $\mathbf{f}=0$, Eq. (1) becomes an eigenvalue

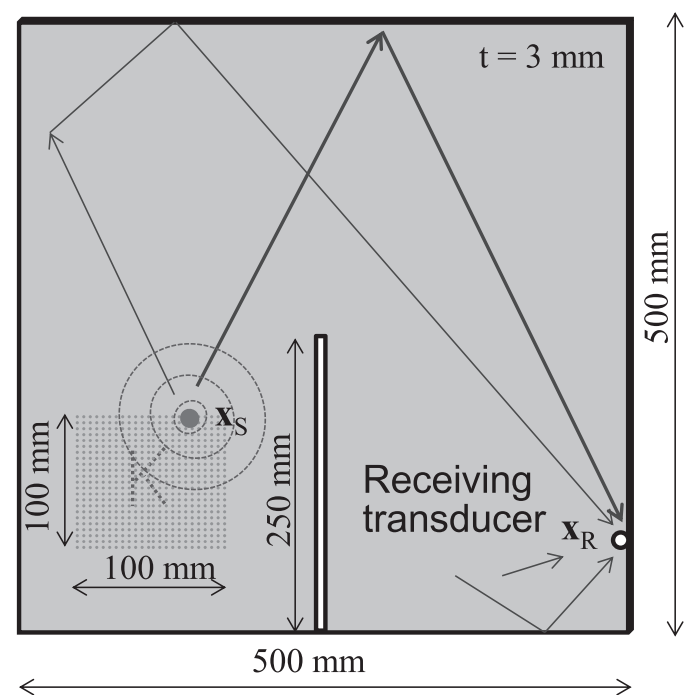

FIG. 1. Specimen used in the present study, and laser source and receiving transducer positions. problem with respect to $\omega$. Letting the eigenvalues be $\omega_{m}$ and the eigenvectors be $\boldsymbol{\varphi}_{m}$, respectively, the solution to Eq. (1) can be written using eigenvectors as

$$
\mathbf{u}=\sum_{m} A_{m} \boldsymbol{\varphi}_{m},
$$

where $A_{m}$ is the amplitude of the $m$ th eigen-mode as determined by the frequency, material geometries, material constants, conditions of incident waves and boundaries, while the eigenvector $\boldsymbol{\varphi}_{m}$ denotes the displacement distribution of the $m$ th mode. Now, considering the characteristic whereby the displacement $\mathbf{u}$ is roughly in inverse proportion to the thickness at the source location $d\left(\mathbf{x}_{S}\right)$ for a dynamic loading in a low $f d$ range, which has been proven in previous studies, ${ }^{14-16}$ the amplitude $A_{m}$ can be substituted into $D\left(\mathbf{x}_{S}\right) A^{\prime}{ }_{m}$, where $D\left(\mathbf{x}_{S}\right) \equiv d_{0} / d\left(\mathbf{x}_{S}\right)$, and $d_{0}$ is the thickness of the intact area. Then, within the limitations of the low $f d$ range, in which the wavelength is much larger than the plate thickness, Eq. (1) approximates to

$$
\mathbf{u} \approx D\left(\mathbf{x}_{S}\right) \sum_{m} A_{m}^{\prime} \boldsymbol{\varphi}_{m} .
$$

Assuming that the defects are sufficiently small and do not affect the displacement distribution of the $m$ th resonant mode $\boldsymbol{\varphi}_{m}$, the term $\sum_{m} A_{m}^{\prime} \boldsymbol{\varphi}_{m}\left(\equiv \mathbf{u}_{\text {intact }}\right)$ is the displacement vector for the case when the laser beam is directed onto a certain point $\mathbf{x}_{S}$ on an intact plate with a uniform thickness. Let the displacement components at the receiving position $\mathbf{x}_{R}$ be $[\mathbf{u}]_{r}$, then the displacement for a laser source $\mathbf{x}_{S}$ at the receiving position $\mathbf{x}_{R}$ is

$$
\left[\mathbf{u}\left(\mathbf{x}_{S}, \omega\right)\right]_{r} \approx D\left(\mathbf{x}_{S}\right)\left[\mathbf{u}_{\text {intact }}\left(\mathbf{x}_{S}, \omega\right)\right]_{r} .
$$

$\left[\mathbf{u}_{\text {intact }}\left(\mathbf{x}_{S}, \omega\right)\right]_{r}$ is the displacement distribution for a range of laser spots $\mathbf{x}_{S}$ in the intact plate with uniform thickness and is affected by multiple reflections at the plate wall and the resonances of the plate. That is, the displacement distribution obtained at the fixed receiving point by a scanning laser source $\mathbf{x}_{S}$ is approximated by the product of the thickness distribution function $D\left(\mathbf{x}_{S}\right)$ and the displacement distribution for the intact plate $\left[\mathbf{u}_{\text {intact }}\left(\mathbf{x}_{S}, \omega\right)\right]$. If these multiple reflections are small, the displacement distribution $\left[\mathbf{u}\left(\mathbf{x}_{S}, \omega\right)\right]_{r}$ represents the defect image corresponding to the thickness distribution $D\left(\mathbf{x}_{S}\right)$.

Next, we experimentally verified that the thickness distribution $D\left(\mathbf{x}_{S}\right)$ can be obtained for a plate with complex geometries in which there are no straight paths between the laser sources and a receiving point. Figure 1 shows the specimen used, the laser source area, and receiving position. An aluminum alloy plate measuring $500 \mathrm{~mm} \times 500 \mathrm{~mm} \times 3 \mathrm{~mm}$ was cut half-way along its centerline to block the direct waves used in Ref. 13, and a letter $\mathrm{K}$ was engraved $1.5 \mathrm{~mm}$ in depth and $2.0 \mathrm{~mm}$ in width behind the left-hand region as an artificial defect. The laser beam was aimed at an area measuring $100 \mathrm{~mm} \times 100 \mathrm{~mm}$ with a $1-\mathrm{mm}$ pitch on the plate surface behind which the artificial defect was located, and the subsequent elastic waves were received by the piezoelectric transducer with a central frequency of about $8 \mathrm{kHz}$, attached to the right-hand edge of the plate surface. Elastic 


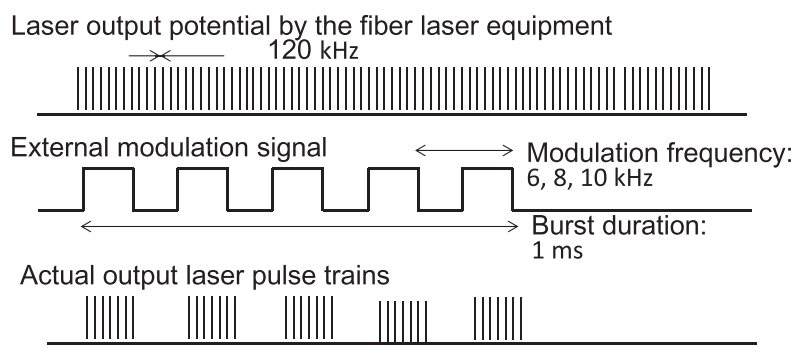

FIG. 2. Modulation signal and laser output used in this study.

waves were generated by a fiber laser that can output laser pulses at a high repetition rate. The output of the fiber laser equipment could be controlled using external modulation signals, as shown in Fig. 2. Narrowband burst waves were generated by the laser output at a repetition rate of $120 \mathrm{kHz}$ modulated by square signals from $6 \mathrm{kHz}$ to $10 \mathrm{kHz}$ with a 1 ms duration. The frequency-controlled laser output enabled the conduction of defect imaging experiments at different frequencies.

Figure 3 shows a waveform and its Fourier spectrum for a certain location of the laser source when $8-\mathrm{kHz}$ modulation signals were applied. The signals received by the piezoelectric transducer were amplified up to $60 \mathrm{~dB}$ by a pre-amplifier, digitized by an analog-to-digital converter, and then filtered by a band-pass filter from 2 to $12 \mathrm{kHz}$ on a personal computer. We can see, in waveform (a), that a large number of wave packets appear after about $1 \mathrm{~ms}$ due to multiple reflections, and almost all of the waves disappear after $20 \mathrm{~ms}$ due to the attenuation in the plate and leakage into the air. The frequency spectrum (b) exhibits a distinct peak at about $8 \mathrm{kHz}$, which proves that frequency components corresponding to the modulation signal were generated appropriately in the experimental system.

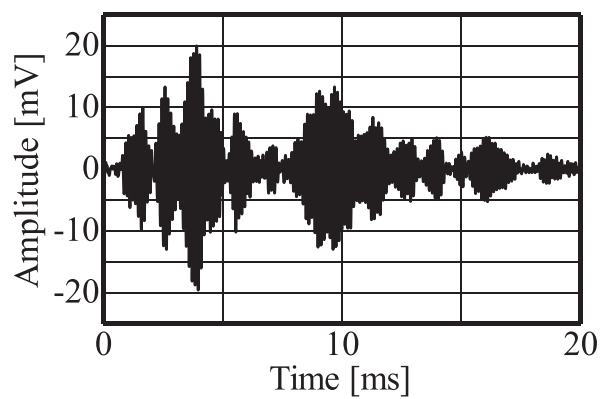

(a)

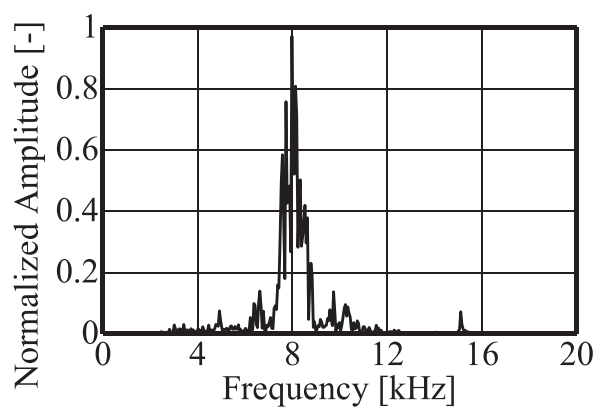

(b)

FIG. 3. Typical waveform (a) and frequency spectrum (b) recorded in the experiment for $8 \mathrm{kHz}$.

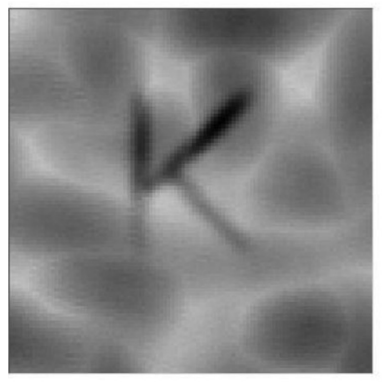

(a)

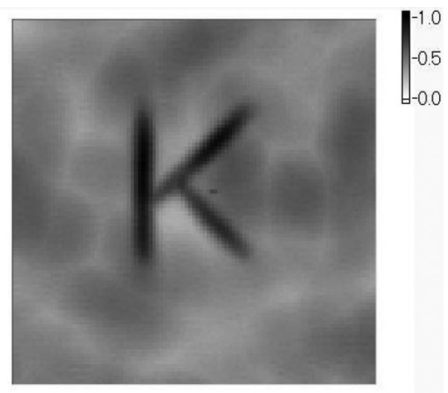

(b)
FIG. 4. Frequency peak distributions. (a) Distribution at a single incident frequency of $8 \mathrm{kHz}\left[\mathbf{u}\left(\mathbf{x}_{S}, \omega\right)\right]_{r}$. (b) Averaged distributions of 6,8 , and $10 \mathrm{kHz}\left[\mathbf{u}_{A V E}\left(\mathbf{x}_{S}\right)\right]_{r}$.

Figure 4(a) is the frequency peak distribution obtained when the laser beam scanned an area measuring $100 \mathrm{~mm} \times 100 \mathrm{~mm}$. As shown in Fig. 2, 8-kHz modulation signals were used and the distribution images were obtained from the peak values of the frequency spectrum between $7 \mathrm{kHz}$ and $9 \mathrm{kHz}$. The artificial defect (the letter $\mathrm{K}$ ) was visible, proving that the defect imaging technique is effective even in a plate with complex geometries and with no direct paths for the elastic waves. However, in Fig. 4(a), the letter $\mathrm{K}$ is partially obscured and non-uniform gray areas exist in the intact region. This is caused by the resonant effect in the plate $\left[\mathbf{u}_{\text {intact }}\left(\mathbf{x}_{S}, \omega\right)\right]_{r}$.

Now, noting that the thickness distribution $D\left(\mathbf{x}_{S}\right)$ is independent of the frequency $\omega$ and the resonant pattern $\left[\mathbf{u}_{\text {intact }}\left(\mathbf{x}_{S}, \omega\right)\right]_{r}$ varies with $\omega$, taking the average of the distributions at different frequencies reduces the resonant pattern to

$$
\left[\mathbf{u}_{A V E}\left(\mathbf{x}_{S}\right)\right]_{r}=D\left(\mathbf{x}_{S}\right) \sum_{n=1}^{N}\left[\mathbf{u}_{\text {intact }}\left(\mathbf{x}_{S}, \omega_{n}\right)\right]_{r} / N
$$

The summation term gradually approaches a uniform distribution as the number of distributions at different frequencies $N$ increases. Distributions at various frequencies $\left[\mathbf{u}\left(\mathbf{x}_{S}, \omega_{n}\right)\right]_{r}$ can now be obtained easily because elastic wave generation with the fiber laser can control the frequency content by changing the modulation signals. Figure 4(b) shows the averaged distribution $\left[\mathbf{u}_{A V E}\left(\mathbf{x}_{S}\right)\right]_{r}$ of three distributions at $6 \mathrm{kHz}$, $8 \mathrm{kHz}$, and $10 \mathrm{kHz}$. The defect image is clear and the resonance patterns at the intact region are also significantly reduced.

Because this imaging technique can be applied to platelike structures, regardless of the distance between the laser source $\mathbf{x}_{S}$ and the receiving position $\mathbf{x}_{R}$ and the propagation paths of elastic waves, the technique will be applicable to a wide variety of applications from large structures such as existing pipes and tanks to thin films and membranes. Moreover, since a laser source is scanned by mirrors, this technique has the potential for extremely fast imaging and can be applied to in-line inspection in production lines.

This work was supported by JSPS KAKENHI Grant No. 26282094 and Chubu Electric Power Co., Inc. 
${ }^{1}$ H. Ammari, E. Bretin, J. Garnier, H. Kang, H. Lee, and A. Wahab, Mathematical Methods in Elasticity Imaging (Princeton University Press, Princeton, 2015).

${ }^{2}$ D. W. Prine, Proceedings of the Engineering Applications of Holography Symposium: February 16-17, 1972, Los Angeles, California (SPIE, 1972), p. 287.

${ }^{3}$ S. R. Doctor, T. E. Hall, and L. D. Reid, NDT Int. 19, 163 (1986).

${ }^{4}$ B. W. Drinkwater and P. D. Wilcox, NDT\&E Int. 39, 525 (2006).

${ }^{5}$ J. L. Rose, Ultrasonic Waves in Solid Media (Cambridge University Press, Cambridge, 1999).

${ }^{6}$ P. Cawley, M. J. S. Lowe, D. N. Alleyne, B. Pavlakovic, and P. Wilcox, Mater. Eval. 61, 66 (2003).

${ }^{7}$ T. Hayashi and M. Murase, J. Acoust. Soc. Am. 117, 2134 (2005).

${ }^{8}$ A. Migliori and J. Sarrao, Resonant Ultrasound Spectroscopy (Wiley, New York, 1997).

${ }^{9}$ H. Ogi, G. Shimoike, M. Hirao, K. Takashima, and Y. Higo, J. Appl. Phys. 91, 4857 (2002).
${ }^{10}$ N. Dominguez, V. Gibiat, and Y. Esquerre, Wave Motion 42, 31 (2005).

${ }^{11}$ N. Dominguez and V. Gibiat, Ultrasonics 50, 367 (2010).

${ }^{12}$ S. Rodriguez, P. Sahuguet, V. Gibiat, and X. Jacob, Ultrasonics 52, 1010 (2012).

${ }^{13}$ S. Rodriguez, M. Deschamps, M. Castaings, and E. Ducasse, Ultrasonics 54, 1880 (2014).

${ }^{14}$ S. Rodriguez, M. Veidt, M. Castaings, E. Ducasse, and M. Deschamps, Appl. Phys. Lett. 105, 244107 (2014).

${ }^{15}$ H. Ammari, J. Garnier, V. Jugnon, and H. Kang, SIAM J. Control Optim. 50, 48 (2012).

${ }^{16}$ H. Ammari, E. Bretin, J. Garnier, W. Jing, and A. Wahab, SIAM J. Imaging Sci. 6, 2174 (2013).

${ }^{17}$ T. Hayashi, M. Murase, and M. N. Salim, J. Acoust. Soc. Am. 126, 1101 (2009).

${ }^{18}$ T. Hayashi, M. Murase, and T. Kitayama, Ultrasonics 52, 636 (2012).

${ }^{19}$ T. Hayashi, M. Murase, N. Ogura, and T. Kitayama, Mater. Trans. 55, 1045 (2014). 